\title{
Prospective Clinical Study of 125 I Particle Permanent Implantation for Prostate Cancer
}

\author{
Yupeng Xin ${ }^{*}$, Daoling Guo², Jiagao Qi², Zhengguo Chen³, Honggang Shao', Guangya Yuan1 \\ ${ }^{1}$ Department of Urology, Mianyang Central Hospital, Mianyang, Sichuan, China \\ ${ }^{2}$ Department of Ultrasound, Mianyang Central Hospital, Mianyang, Sichuan, China \\ ${ }^{3}$ Department of Nuclear Medicine, Mianyang Central Hospital, Mianyang, Sichuan, China \\ Email: *xinyupeng71@163.com
}

How to cite this paper: Xin, Y.P., Guo, D.L., Qi, J.G., Chen, Z.G., Shao, H.G. and Yuan, G.Y. (2020) Prospective Clinical Study of ${ }^{125}$ I Particle Permanent Implantation for Prostate Cancer. Open Journal of Urology, 10, 52-59.

https://doi.org/10.4236/oju.2020.103007

Received: December 13, 2019

Accepted: February 18, 2020

Published: February 21, 2020

Copyright (c) 2020 by author(s) and Scientific Research Publishing Inc. This work is licensed under the Creative Commons Attribution International License (CC BY 4.0)

http://creativecommons.org/licenses/by/4.0/

Open Access

\begin{abstract}
Objective: To investigate the clinical effect of ${ }^{125} \mathrm{I}$ radioactive seed implantation and the treatment of prostate cancer with radical resection of prostate cancer. Methods: Within the period of the second phase, 62 cases of prostate cancer patients, aged from 46 to 87 years old, average 69 years old. In the treatment group, 30 cases were implanted with ${ }^{125} \mathrm{I}$ radioactive particles via the rectum with the guidance of the rectum. 32 cases in the control group underwent radical prostatectomy. Results: All patients were operated successfully, the patients were followed up for 12 to 36 months. In the treatment group, the average particle size was $38+15$. No serious complications caused by rectal puncture. 2 patients occurred 6 months after PSA increased significantly, systemic examination revealed bone metastases, and underwent endocrine therapy. In the control group, there were 2 cases of patients with PSA significantly increased in 5 months after surgery, and the external irradiation plus endocrine therapy. During the observation period, the survival rate of the patients who were implanted with ${ }^{125} \mathrm{I}$ particles in the treatment group without progression was $93.3 \%$. Control group, the cumulative PSA progression free survival rate was $93.7 \%$. Conclusion: Patients with prostate cancer during the second phase, the clinical curative effect of the treatment of prostate cancer with the treatment of ${ }^{125} \mathrm{I}$ of prostate cancer and the treatment of prostate cancer by using radical resection of prostate cancer are quite. This technology has the advantages of small trauma, quick recovery, low damage to normal tissue.
\end{abstract}

\section{Keywords}

Transrectal ${ }^{125}$ I Seeds Implantation, Prostate Cancer, Internal-Radiation Therapy, Transrectal Ultrasonography 


\section{Introduction}

In recent years, the incidence of prostate cancer is increasing year by year, which has become a threat to the health of male reproductive system of the main diseases, Brachytherapy for the treatment of prostate cancer is a form of treatment for prostate cancer, and is widely used in the treatment of prostate cancer. Near distance treatment is the use of special equipment, under the guidance of CT or B ultrasound, through a special guidance system will be directly put into the prostate, radioactive radionuclides continue to release radiation to kill the tumor cells. Compared with radical prostatectomy and external radiation therapy (EBRT), brachytherapy has the same biochemical control rate, and complications are more likely to be accepted by patients. There was no significant difference in the 5 -year survival rates between brachytherapy and radical prostatectomy [1]. As a form of radiation therapy, the complications and the quality of life of patients, and the patient's tolerance degree have obvious advantages, therefore, more and more attention has been paid to brachytherapy. From March 2006 to May 2013, our hospital used ${ }^{125}$ I radioactive seed implantation in the treatment of 30 cases of prostate cancer in the second phase, and 32 patients with prostate cancer underwent radical surgery during the same period as a control study. Each patient who participated in the clinical study signed a declaration of voluntary participation in the clinical study, the study was also approved by the hospital ethics committee in order to evaluate the effect of the treatment. Now the report is as follows.

\subsection{General Information}

From March 2006 to May 2013, 62 cases of prostate cancer were treated in our department. The age ranged from 46 to 87 years, with an average of 69 years. Before surgery, the prostate biopsy was proved to be prostate cancer, Gleason score of 4 to 7 points, PSA was detected between 5.82 to $53.6 \mathrm{ng} / \mathrm{ml}$, with an average of $23.6 \mathrm{ng} / \mathrm{ml}$. All patients were diagnosed by rectal examination, rectal prostate ultrasound, prostate MRI, chest X-ray, bone scan and PSA examination, TNM staging (2002AJCC) was $\mathrm{T}_{2} \mathrm{~N}_{0} \mathrm{M}_{0}$. In the treatment group, 30 cases were treated with ${ }^{125}$ I radioactive seed therapy by the rectum ultrasound-guided prostate implantation. In the control group, 32 cases underwent radical prostatectomy, including 21 patients underwent open radical prostatectomy, 11 cases underwent laparoscopic radical resection of the prostate cancer.

\subsection{Instrument}

MINDRAY DC-6 and SSD-350A Toshiba color ultrasound diagnostic instrument, dual purpose probe $(5-7.0 \mathrm{MHz})$ and corresponding puncture rack, Treatment planning system of Zhuhai hokai medical company (TPS) and rotary gun implantation. Shanghai Xinke Medicines Co ${ }^{125} \mathrm{I}$ particles $(0.7-0.8 \mathrm{mCi}$ ), with eight PTC light implantation needle puncture needle $18 \mathrm{G}$. 


\subsection{Method}

\subsubsection{Particle Implantation Method}

Using ultrasonography transrectal (TRUS) from the bottom of the prostate to the tip of the tip of each septum $0.5 \mathrm{~cm}$ acquisition of a cross-sectional image input TPS 3D reconstruction, Get the prostate and its surrounding tissue profile and position, and then get the volume of the prostate $\left(\mathrm{T} 1-2 \mathrm{~N}_{0} \mathrm{M}_{0}\right.$ to obtain the true volume of the prostate) about $17.1-81.2 \mathrm{ml}$, average $(40.0+16.2) \mathrm{ml}$. The middle enlargement in 53 cases, 42 cases of protrusion protruding into the bladder, volume $8.4(1.0$ - 23.1) $\mathrm{ml}$. According to the American Brachytherapy Society (ABS) standardized program outline plan target area [2]. Target diameter line and ${ }^{125}$ I activity preset interparticle spacing and particle and target edge spacing according to the preset plane implantation for longitudinal section, a contact pin cloth by forward, according to the ABS recommended standard [2] set prescription dose 145 Gy obtained particle space distribution, number and dose response curves etc. The modified method was used to adjust the distribution of particles and the number of particles, the control of the quantity of the urethra was less than 2 times of the prescription dose of [2], and the rectal dose was controlled from $100 \%$ to $120 \%$ [3], To cover the target area with the $100 \%$ dose curve, thereby obtaining a treatment plan. Preoperative 3D quinolone antibiotics, preoperative cleansing enema, catheter placement. Without anesthesia, right lateral decubitus, song of the hip and knee, disinfects shop towel, puncture mounting bracket, TURS is once again in full scan check the prostate and surrounding tissue to the approved treatment plan. In the longitudinal section of the implementation of puncture and implantation, by the urethra of the implant level (the middle lobe increases) or near the level of both sides of the urethra began to layer by layer outwards, each level are used from the bottom to the top of the order (see Figure 1). Less postoperative activity, at least $3000 \mathrm{ml}$ daily drinking water, preventive anti-infection and hemostatic treatment.

In the control group, 32 cases underwent radical prostatectomy, including 21 cases underwent open radical prostatectomy, 11 cases underwent laparoscopic radical resection of the prostate cancer. Laparoscopic radical prostatectomy was performed in patients with laparoscopic surgery, and open surgery group underwent conventional open radical prostatectomy. The basic process of surgery: The left pelvic fascia is separated from the anterior wall of the bladder and the two side walls of the bladder, and the left pelvic fascia is separated, and the prostate is pushed away from the side direction of the anus to the side direction of the prostate, and the right side and the back side of the prostate are separated by the same method. Suture ligation of the penile dorsal vein complex, ultrasound knife to cut off the ligament of the prostate, suture penile dorsal vein complex. Off the neck of the bladder. Free seminal vesicle and prostate back. Transection of the urethra and free prostate, complete resection of the prostate. Anastomosis of bladder neck and urethra. Take out the 


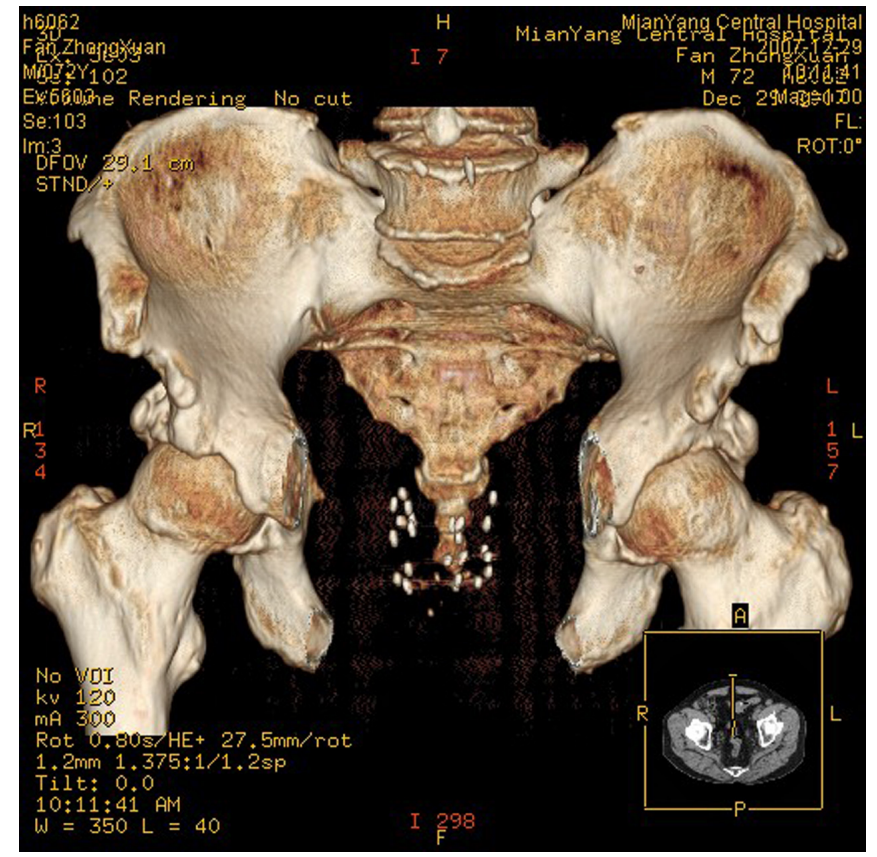

Figure 1. Prostate cancer patients with rectal prostate implanted ${ }^{125}$ I radioactive particles after the pelvic CT review pictures.

specimen and suture the incision.

\subsection{Follow up Method}

From first days after the start of after operation, observed in June 2015, every 1 to 3 months to review the PSA, and to understand the micturition function and rectal irritation symptoms, biochemical recurrence of the standard PSA to achieve the lowest value after 3 consecutive rise.

\subsection{Statistics Processing}

SPSS12.0 statistical software was used for statistical analysis, measurement data using t-test, count data using chi-square test, $\mathrm{P}<0.05$ for the difference was significant.

\section{Result}

\subsection{Comparison of Therapeutic Effect and Cost of Hospitalization}

The operative time, blood loss, postoperative hospital stay, blood transfusion and hospitalization expenses of the ${ }^{125}$ I particles implantation in the treatment group were less than that of the prostate cancer radical operation group, Rectal radiation ${ }^{125} \mathrm{I}$ particles implantation in the treatment group was higher than the patients with prostate cancer radical surgery group (see Table 1).

\subsection{Comparison of Major Postoperative Complications between the Two Groups}

The incidence of erectile dysfunction and urinary incontinence in the treatment 
group was lower than that in the radical prostatectomy group, and the incidence of urinary retention in the treatment group was higher than that in the radical prostatectomy group (see Table 2).

\subsection{The Comparison of the Two Groups after Surgery}

All patients were successfully operated, the patients were followed up for 12 to 36 months, and the median follow-up was 28.5 months. The treatment group was $38 \pm 15$ particles. 2 patients occurred 6 months after PSA increased significantly, systemic examination revealed bone metastases, and underwent endocrine therapy. In the control group, there were 2 patients who had a significant increase of PSA in 5 months after operation. Cumulative PSA progression free survival rate was $93.3 \%$ (compared with control group $P>0.05$ ) in the patients who were treated by rectal radioactive ${ }^{125} \mathrm{I}$ seed implantation. Prostate cancer radical surgery group cumulative PSA progression free survival rate was $93.7 \%$.

\section{Discussion}

Prostate cancer is the second most common cancer in the United States. The average annual incidence increased by $8 \%$. Prostate cancer research abroad is very wide, more than 26,000 people in the United States each year to receive radiation therapy. The application of Perineal ultrasound-guided particle implantation in the treatment of prostate cancer is very rapid, and has become one of the standard treatments for early prostate cancer. In recent years, with the change of living environment, diet structure and life style, the incidence of prostate cancer in China has increased year by year, but we still have a great gap with foreign countries in this field. It was not until 2001 that our country was the first to complete the first ${ }^{125} \mathrm{I}$ of the interstitial brachytherapy for prostate cancer. In recent years, the perineal template and TRUS guided percutaneous implantation of particles, positioning accuracy, good reproducibility, and has been widely used. However, the pathway to by puncture, in the larger prostatic (equal to or more than $60 \mathrm{ml}$ ) or pubic arch is too narrow, the puncture path susceptible to pubic

Table 1. Effect of operation and cost of hospitalization $(\bar{X} \pm S D)$.

\begin{tabular}{cccccc}
\hline & $\begin{array}{c}\text { Operation time } \\
\text { (min) }\end{array}$ & $\begin{array}{c}\text { Bleeding during } \\
\text { operation }(\mathrm{ml})\end{array}$ & $\begin{array}{c}\text { Postoperative hospital } \\
\text { stay (day) }\end{array}$ & $\begin{array}{c}\text { Amount of blood } \\
\text { transfusion }\end{array}$ & $\begin{array}{c}\text { Satisfaction } \\
\text { degree }\end{array}$ \\
\hline${ }^{125}$ I particle group & $60.5 \pm 30.2$ & $30.0 \pm 15.0$ & $4.2 \pm 1.5$ & 0 & $7.5 \pm 1.8$ \\
Radical operation group & $195.5 \pm 50.0^{*}$ & $400.0 \pm 120.0^{*}$ & $8.5 \pm 1.6^{*}$ & $380.0 \pm 150.0^{*}$ & $5.8 \pm 2.0^{*}$ \\
\hline
\end{tabular}

${ }^{*} \mathrm{P}<0.01$.

Table 2. Comparison of major postoperative complications between the two groups.

\begin{tabular}{rccc}
\hline & urinary retention & Erectile dysfunction & Urinary incontinence \\
\hline${ }^{125}$ I particle group & $4.2 \pm 2.5$ & $12.0 \pm 3.4$ & 0 \\
Radical operation group & $1.3 \pm 1.2^{*}$ & $17.6 \pm 3.8^{*}$ & $8 \pm 2.6^{*}$ \\
\hline${ }^{*}<0.01$. & & &
\end{tabular}


arch partial occlusions caused prostate seed leakage graft and implant location offset [4], To the middle of the larger easily urethral injury. With middle leaf protruding into the bladder to easily penetrate into the bladder, cause urethral, bladder injury bleeding and particle typographical error [5] [6], these are ABS as a relative contraindication [2]. TRUS guided by the rectal route, the Department of the Department by the forward puncture, to avoid the perineal approach to these aspects of the problem [5] [6]. Operation will not be affected by the pubic arch interference, not easy to hurt the bladder and ureter, the middle of the puncture is not affected by the urethra. No anesthesia was used in the operation of the rectal route and no severe pain. The patients were treated with the ${ }^{125} \mathrm{I}$ radioactive seed implantation comparison of visible with open surgery and the way of perineal puncture. Rectal prostate ${ }^{125}$ I radioactive seed implantation treatment and the use of radical surgery for prostate cancer compared to the operation is more simple and fast, the treatment group ${ }^{125}$ I radioactive particles by rectal implantation average of $60 \mathrm{~min}$ minutes or so, far less than the operation of the control group. The amount of blood loss, postoperative hospital stay, blood transfusion and hospitalization expenses were less than that of prostate cancer radical operation group by the rectum ultrasound-guided ${ }^{125}$ I radioactive particles. The patients' satisfaction rate was higher than that in the radical operation group. No serious complications were caused by rectal puncture. On the comparison of major postoperative complications, the incidence of urinary retention in the treatment group was higher than that of the control group $(\mathrm{P}<0.01)$, analysis of the causes and the anterior prostate volume, increase in the middle lobe, IPSS score, the number of puncture needles, the number of implanted particles $(\mathrm{P}<0.01)$, and age, clinical stage, preoperative Gleason score, PSA independent $(\mathrm{P}$ all $>0.05)$. Ohashi [7] Toshio's study suggests that even after a radioactive dose of tissue is small, there is still an acute retention of urine, so that the cause of retention of urine is not the amount of radioactive material, but the damage to the prostate tissue caused by the implantation of the particles. IPSS is the international prostate symptom score, the higher the number of symptoms of difficulty in urination, the higher the value of the score, the more obvious symptoms of the relative difficulty of urination after implantation, the greater the possibility of acute urinary retention. According to our study, the number of puncture needles and the IPSS score of the implanted particles can be used as a predictive factor for the occurrence of acute urinary retention [8]. Erectile dysfunction (ED) and urinary incontinence in the treatment group were significantly lower than that of the prostate cancer radical operation group after the operation. ED after prostate brachytherapy (PB) is not very clear, but some studies have indicated that ED may be related to the injury of the puncture needle after treatment; After treatment in the long-term process of ED may with the local short distance radiotherapy induced vascular injury, the bulb of penis nerve damage (to reduce the number of neural nitric oxide synthase), thus on penile corpus cavernosum smooth muscle function caused by damage [9]. In the com- 
parison of curative effect after operation in the two groups. After rectal radioactive ${ }^{125} \mathrm{I}$ seeds implantation in the treatment group, 2 cases of patients after 6 months appear PSA is apparently elevatory, systemic examination found bone metastases, underwent endocrine therapy are still alive. Prostate cancer radical surgery group also has 2 cases of patients with PSA significantly increased 5 months after surgery, systemic examination did not find bone metastases, and the external irradiation plus endocrine therapy. Cumulative PSA progression free survival rate was $93.3 \%$ (compared with control group $\mathrm{P}>0.05$ ) in the patients who were treated by rectal radioactive ${ }^{125} \mathrm{I}$ seed implantation. Prostate cancer radical surgery group cumulative PSA progression free survival rate was 93.7\%. For patients with stage II prostate cancer, the treatment of prostate cancer by ${ }^{125} \mathrm{I}$ particles with a diameter of the rectum compared with the radical operation of prostate cancer, the clinical effect is equivalent $(P>0.05)$.

\section{Conclusion}

To Summarize our research results, TRUS guided percutaneous prostate ${ }^{125} \mathrm{I}$ particle implantation puncture complications less, the use of rectal two-dimensional ultrasound and TPS in the development of real-time treatment plan, can accurately reconstruct the three-dimensional shape of the prostate. Design the implantation path, location and quantity of particles to achieve three-dimensional conformal radiotherapy. And the method of the surrounding tissue injury is small, the operation is simple and rapid, safe and feasible, the treatment cost is relatively low, postoperative recovery is fast, no serious complications can improve the survival rate of patients, and contribute to the control of tumors in this type of patients, it is worth clinical application.

\section{Funding}

China Sichuan provincial health department scientific research project fund (number 070202).

\section{Conflicts of Interest}

The authors declare no conflicts of interest regarding the publication of this paper.

\section{References}

[1] Taira, A.V., Merrick, G.S., Butler, W.M., et al. (2011) Long-Term Outcome for Clinically Localized Prostate Cancer Treated with Permanent Interstitial Brachytherapy. International Journal of Radiation Oncology Biology Physics, 79, 1336-1342. https://doi.org/10.1016/j.ijrobp.2010.01.005

[2] Nag, S., Beyer, D., Friedland, J., et al. (1999) American Brachytherapy Society Recommendations for Transperineal Permanent Brachytherapy of Prostate Cancer. International Journal of Radiation Oncology Biology Physics, 44, 789-799. https://doi.org/10.1016/S0360-3016(99)00069-3

[3] Merrick, G.S., Butler, W.M., Dorsey, A.T., et al. (2000) Rectal Function Following 
Prostate Brachytherapy. International Journal of Radiation Oncology Biology Physics, 48, 667-674. https://doi.org/10.1016/S0360-3016(00)00698-2

[4] Sugawara, A., Nakashima, J., Shigematsu, N., et al. (2009) Prediction of Seed Migration after Transperineal Interstitial Prostate Brachytherapy with I-125 Free Seeds. Brachytherapy, 8, 52-56. https://doi.org/10.1016/j.brachy.2008.10.003

[5] Guo, D.N., Gao, J.G., W, D., et al. (2009) Trans Rectal Ultrasound Guided Trans Rectal Implantation and Its Application in the ${ }^{125}$ I Particle Implantation of Prostate Cancer. Chinese Journal of Ultrasound Medicine, 25, 980-982.

[6] Wu, H.M., Lv, J., Hu, W., et al. (2013) The Clinical Effect of ${ }^{125}$ I Seeds Implantation Combined with Endocrine Therapy in the Treatment of Locally Advanced Prostate Cancer. Chinese Journal of Male, 19, 617-621.

[7] Toshio, O., Atsunori, Y., Kazuhito, T., et al. (2006) Predictive Factors of Acute Urinary Retention Requiring Catheterization Following ${ }^{125}$ I Prostate Brachytherapy. Japanese Journal of Clinical Oncology, 36, 285-289. https://doi.org/10.1093/jjco/hyl008

[8] Shao, H.G., Yuan, G.Y., Guo, D.N., et al. (2012) Multi Factor Analysis of Acute Urinary Retention in Patients with Prostate Cancer after ${ }^{125} \mathrm{I}$ Brachytherapy. Sichuan Medical Journal of China, 33, 1109-1111.

[9] Akbal, C., Tinay, I., Simsek, F., et al. (2008) Erectile Dysfunction Following Radiotherapy and Brachytherapy for Prostate Cancer: Pathophysiology, Prevention and Treatment. International Urology and Nephrology, 40, 355-363.

https://doi.org/10.1007/s11255-007-9247-1 